\title{
What Matters in Neuronal Locking?
}

\author{
Wulfram Gerstner \\ Physik-Department der TU München, D-85747 Garching bei München, Germany \\ J. Leo van Hemmen \\ Physik-Department der TU Mïnchen, D-85747 Garching bei München, Germany* \\ Department of Mathematics, University of Chicago, Chicago, IL 60637 USA
}

Jack D. Cowan

Department of Mathematics, University of Chicago, Chicago, IL 60637 USA

Exploiting local stability, we show what neuronal characteristics are essential to ensure that coherent oscillations are asymptotically stable in a spatially homogeneous network of spiking neurons. Under standard conditions, a necessary and, in the limit of a large number of interacting neighbors, also sufficient condition is that the postsynaptic potential is increasing in time as the neurons fire. If the postsynaptic potential is decreasing, oscillations are bound to be unstable. This is a kind of locking theorem and boils down to a subtle interplay of axonal delays, postsynaptic potentials, and refractory behavior. The theorem also allows for mixtures of excitatory and inhibitory interactions. On the basis of the locking theorem, we present a simple geometric method to verify the existence and local stability of a coherent oscillation.

\section{Introduction}

Coherence may be defined as being "united in relationship" for most vertebrate neurons, meaning a temporal relationship in that they fire in unison. As such, it is another way of saying that neurons get locked. Once the proposal appeared that coherent oscillations may exist in biological neural systems (Eckhorn et al. 1988; Gray and Singer 1989; Gray et al. 1989; Engel et al. 1991a, 1991b; Eckhorn et al. 1993; Gray 1994), locking phenomena attracted a considerable amount of interest and spurred quite a few people to explain or disprove the very existence of coherent oscillatory activity. Different authors have used differing models, which vary in several aspects, as do the assumptions and the results. Some models show perfect locking, others partial locking or no locking at all. Some use excitatory interactions, some exploit inhibitory ones, and others use a mixture. In this paper, we present a unifying framework that allows one to derive exact conditions for the existence and stability of coherent

*permanent address

Neural Computation 8, 1653-1676 (1996) (C) 1996 Massachusetts Institute of Technology 
solutions in a network of spiking neurons and to isolate the neuronal characteristics that are essential to them. The result is surprisingly simple: Perfect locking is possible only if firing occurs while the contribution evoked by incoming pulses (i.e., the postsynaptic potentials) is increasing in time. A more precise formulation is given in the next section, where we show how a subtle interplay of axonal delays, postsynaptic potentials, and refractory behavior can lead to coherence. This result can be applied to excitatory or inhibitory couplings or homogeneous mixtures thereof and solves the often-posed question of whether excitation or inhibition is "more suitable" to support collective oscillations (van Vreeswijk et al. 1994; Lytton and Sejnowski 1991). In fact, for spiking neurons, this kind of collective behavior seems to be generic. Furthermore, we present a purely geometric method to verify whether a coherent oscillation can exist and, if so, whether it is stable. In view of the truly extensive and diverse literature, we think a unifying framework meets an urgent need.

In this paper, we concentrate on analytic results for model networks of spiking neurons (Mirollo and Strogatz 1990; Kuramoto 1991; Gerstner and van Hemmen 1992, 1993; Gerstner et al. 1993; Abbott and van Vreeswijk 1993; Bauer and Pawelzik 1993; Tsodyks et al. 1993; Treves 1993; Usher et al. 1993; van Vreeswijk et al. 1994; Gerstner 1995; Ernst et al. 1995; Hansel et al. 1995). We mostly focus on large networks, although our technique can also be applied to small sets of neurons such as central pattern generators (cf. Skinner et al. 1994). We neither consider phase models (Abbott 1990; Schuster and Wagner 1990a; Sompolinsky et al. 1990; Niebur et al. 1991; Golomb et al. 1992) nor analyze simulation studies (Buhmann 1989; Bush and Douglas 1991; Lytton and Sejnowski 1991; Schuster and Wagner 1990b; König and Schillen 1991; Schillen and König 1991; von der Malsburg and Buhmann 1992; Engel et al. 1992; Deppisch et al. 1993; Nischwitz and Glünder 1995; Ritz et al. 1994). Furthermore, we do not comment on the debate concerning the interpretation and potential relevance of coherent states since there are already many papers arguing the issue (Eckhorn et al. 1988; Gray et al. 1989; Engel et al. 1991a; Schuster and Wagner 1990b; König and Schillen 1991; von der Malsburg and Buhmann 1992; Ritz et al. 1994. Cf. in particular von der Malsburg 1994; von der Malsburg and Schneider 1986; Singer 1994).

In order to prove our locking result, we will use the framework of the spike response model (Gerstner 1991; Gerstner and van Hemmen 1992, 1993; Gerstner 1991, 1995; Kistler et al. 1996). In this model, the effects of spike emission and spike reception are described by two response kernels: 1, to represent a spike and the resulting refractory behavior, and $\therefore$ to take into account the response of a neuron once a spike has arrived at a synapse on its dendritic tree. If a presynaptic neuron $j$ fires at a time $t_{i}^{f}$, a response will be evoked at the soma of a postsynaptic neuron $i$, which we describe by $J_{i j} \equiv\left(t-t_{i}^{f}\right)$. The synaptic weight $J_{i j}$ is a measure of the amplitude of the response. Similarly, if the neuron $i$ fires at a 
time $t_{i}^{f}$, the repolarization after the pulse usually causes a sharp drop of the membrane potential. This effect is summarized by an additive contribution $\eta\left(t-t_{i}^{f}\right) \leq 0$ to the membrane potential. Typical examples of $\varepsilon$ and $\eta$ can be found in Figures $1 \mathrm{a}$ and $1 \mathrm{~b}$, whereas a more elaborate structure is shown in Figures $1 \mathrm{c}$ and $1 \mathrm{~d}$. A neuron model is said to have a standard dynamics if $d \eta / d s \geq 0$ for all $s>0$. This includes integrate-andfire, fast spiking, and adaptive neurons but excludes intrinsic bursters (cf. Connors and Gutnick 1990 for a classification of neuronal firing patterns). A neuron model with $\eta(s)=\varepsilon(s)=0$ for $s \geq 2 T$ will be called a model with short-term memory. Here $T$ is the period of a network oscillation, to be studied below.

For the sake of simplicity we will assume throughout this paper that the delay $\Delta_{i j}$ between neuron $j$ and neuron $i$ depends on neither $i$ nor $j$. Hence $\Delta_{i j}=\Delta$ and the delay can be incorporated in the function $\varepsilon$. The total membrane potential at the soma of neuron $i$ can then be written

$$
h_{i}(t)=\sum_{f} \eta\left(t-t_{i}^{f}\right)+\sum_{j} J_{i j} \sum_{f} \varepsilon\left(t-t_{j}^{f}\right) .
$$

Due to causality, we have $\eta(s)=0$ for $s<0$ and $\varepsilon(s)=0$ for $s<\Delta$ (cf. Fig. 1a-c). A neuron fires once its membrane potential $h(t)$ reaches a threshold $\vartheta$ from below. This condition defines the firing times $t_{i}^{f}$ and is at the basis of our formalism. For the moment we do not include noise so as to simplify the ensuing arguments even further.

Before turning to the proof of our locking theorem in Section 4, we illustrate its potentialities by presenting a purely geometric method to construct and verify the stability of a coherent oscillation in Section 2 . We indicate the relation between the present setup and the usual integrateand-fire models in Section 3. With respect to locking, it hardly makes any difference whether one uses excitatory or inhibitory couplings. As we will show in Section 2, the geometric method makes such a statement obvious. In Section 5 we return to this fact, which at first sight is surprising, and summarize our findings.

\section{Geometric Method}

In Section 4 we will prove a locking theorem, which is instrumental to understanding neuronal coherence. In this section we take it as the starting point of a purely geometric method that allows one to construct and directly verify the stability of a coherent oscillation. Here is a theorem that relates neuronal characteristics to asymptotic stability, that is, when perturbations of a limit state decay to zero. Most of the time we will simply say that something is stable, meaning that it is asymptotically stable. Precise conditions and extensions will be spelled out in the next section. 
a)

b)

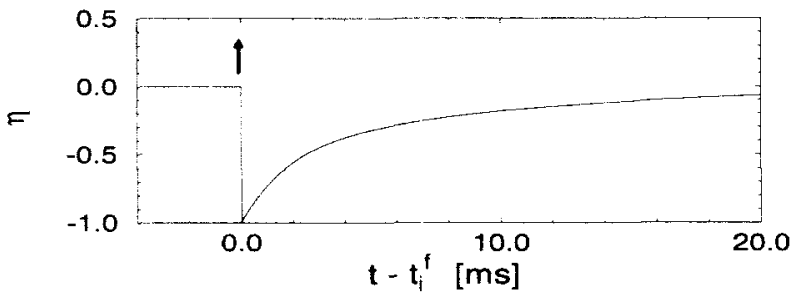

c)
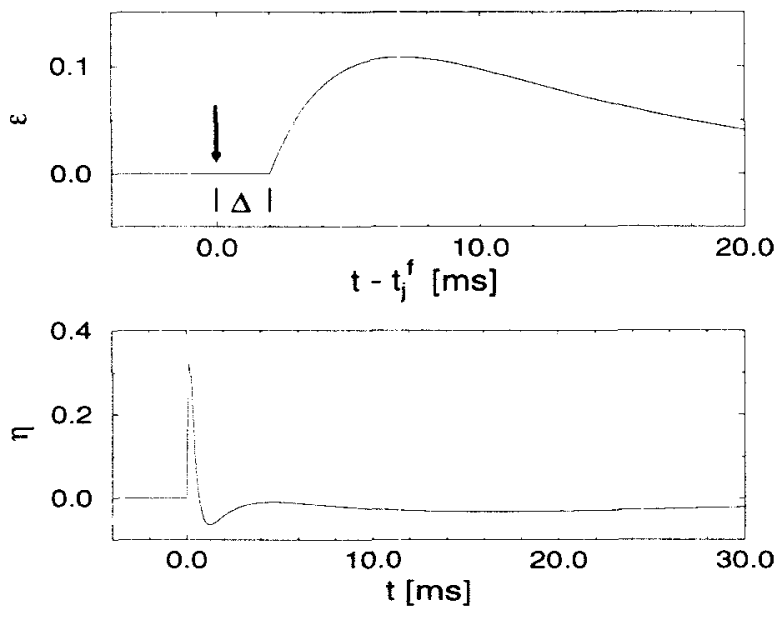

d)

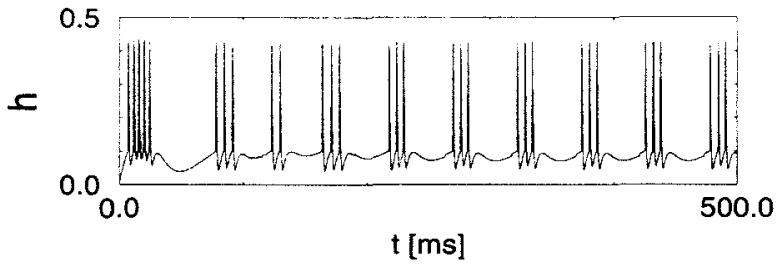

Figure 1: Typical response kernels. (a) Refractory kernel $\eta$. The spike generated at time $t_{i}^{f}$ is indicated by the arrow. After the spike, there is a period of hyperpolarization that decays over $20 \mathrm{~ms}$. (b) Response kernel $\triangleq$. The graph with $s=t-t_{i}^{i}$ exhibits the typical time course of an excitatory postsynaptic potential that is evoked with a delay $\Delta=2 \mathrm{~ms}$ after a presynaptic spike of neuron $j$ at time $t=t_{i}^{f}$ (arrow). The response has been taken at neuron $i$. For $s>\Delta$, we have plotted the function $\Xi(s)=\exp \left[-(s-\Delta) / \tau_{m}\right]\left\{1-\exp \left[-(s-\Delta) / \tau_{\text {syn }}\right]\right\}$ representing a postsynaptic potential for excitatory synaptic input with synaptic time constant $\tau_{\mathrm{svn}}=4 \mathrm{~ms}$ and membrane time constant $\tau_{m}=10 \mathrm{~ms}$. (c) A more elaborate refractory kernel (with four different time constants referring to four different ion channels) gives rise to intrinsic bursting (d), which is a direct consequence of the subsequent hyperpolarization and depolarization exhibited by $\%$. In (d), a neuron with threshold $\theta=0.1$ receives a constant input current. The membrane voltage has been given in arbitrary units. 


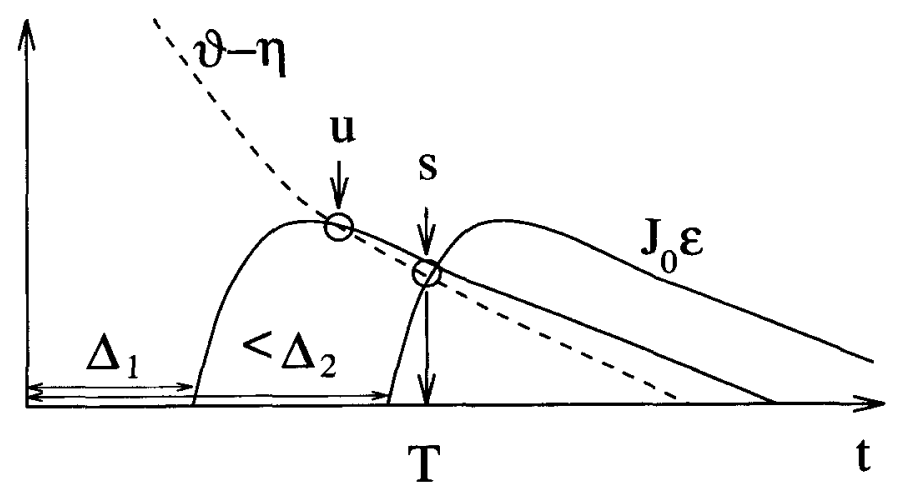

Figure 2: Geometric method: Excitatory couplings. All active neurons have fired at $t=0$. The next spike occurs if $J_{0} \varepsilon(t)$ (solid line) crosses the decreasing threshold $\vartheta-\eta(t)$ (dashed). We have sketched two situations: short $\left(\Delta_{1}\right)$ and long delay $\left(\Delta_{2}>\Delta_{1}\right)$. The coherent oscillation is stable for excitatory couplings with relatively long delays but not for short delays; stable and unstable have been denoted by $(s)$ and $(u)$, respectively.

Locking theorem. In a spatially homogeneous network of spiking neurons with standard dynamics, a necessary and, in the limit of a large number $\mathrm{n}$ of presynaptic neurons $(n \rightarrow \infty)$, also sufficient condition for a coherent oscillation to be asymptotically stable is that firing occurs when the postsynaptic potential arising from all previous spikes is increasing in time.

Let us now turn to Figure 2. The horizontal axis is the time axis, and the vertical axis displays the response of a "typical" neuron. The network under consideration has excitatory interactions only. Each neuron has short-term memory and receives input from $n \gg 1$ other neurons through synaptic weights $J_{0} / n$; the normalization by $1 / n$ is just convenient. We suppose that all neurons fire at time $t=0$. Each neuron then feels its refractory field $\eta$. The action potentials have disappeared into the axons, but after a delay of $\Delta \mathrm{ms}$ they reappear at the dendritic trees and induce a response at the soma, which is described by the function $\varepsilon$. If the postsynaptic potential at the soma reaches the threshold $\vartheta$ of the neuron, so that $\left(J_{0} / n\right) n \times \varepsilon(s)+\eta(s)=\vartheta$ or, equivalently, $J_{0} \varepsilon(s)=\vartheta-\eta(s)$, then all the neurons will fire again. This leads to a simple graphic solution for $T$. As is evident from the plot, in firing again, a neuron still feels its refractory field. If the delay $\Delta$ is too short, the point of intersection of $\varepsilon(s)$ and $\vartheta-\eta(s)$ is in the descending part of $\varepsilon$, and no stable oscillation can arise. If, however, $\Delta$ is a bit longer, then the point of intersection of the two curves is in the ascending part of $\varepsilon$, and a coherent oscillation 
is stable. Once we know the locking theorem, existence and stability can indeed be verified geometrically.

The inhibitory case of Figure 3 does not provide any additional difficulty. It is plain that, to get a response from this purely inhibitory system, we need a stimulus $I_{0}>0$. Again we suppose that all (possibly selected) neurons fire at time $t=0$. Of course, each neuron feels its refractory field 1\% The action potentials disappear into the axons, but after a delay of $\Delta$ ms they reappear at the dendritic trees and induce a response at the soma via the function $z^{\text {inh }}$, which is now negative. The neurons will fire again, provided $\left.J_{0} \varepsilon^{\text {inh }}(s)+I_{0}=1\right)-\eta(s)$. For small $\triangle$ 's or short-lived inhibitory potentials, the neuron still notices its refractory past and the point of intersection is in the ascending part of $z^{\text {inh }}$ (Fig. 3a). If the delay lasts long enough, then " plays no role any more (Fig. 3b), and we are left with the condition $\left.I_{0}+J_{0}{ }^{i n h}(s)=1\right)$ and, hence, stability. In the presence of mere inhibition, the oscillation is stable for a wide range of delays $\Delta$-in contrast to the excitatory case, where the stability depends critically on $\Delta$. Systems with both excitatory and inhibitory interactions are in general more interesting from a neurobiological point of view and will be treated in Section 5. Though it is a simple matter to play around with delays and parameters, we will not pursue this issue here and turn instead to the mathematics of our locking argument. Before delving into the details of the proof, whose geometric essence can be found in Figure 4, we quickly indicate the relation between the usual integrate-and-fire models and the spike response model as it is employed in this paper.

\section{Relation to Integrate-and-Fire Models}

In integrate-and-fire models, firing leads to an immediate reset of the membrane potential. We denote the membrane potential of an integrateand-fire neuron by $\bar{h}(t)$ and its threshold by $\tilde{i}$. Firing occurs if $\tilde{h}(t)=\tilde{v}$. This defines a firing time $t_{i}^{f}$ and the reset requirement is

$$
\lim _{b \rightarrow 0} \tilde{h}\left(t_{i}^{f}+\phi\right)=0 \text {. }
$$

Between two firings, the change of the membrane potential is given by the equation of a simple $R C$ circuit charged by a current $I_{0}+I_{i}(t)$,

$$
\frac{d}{d t} \tilde{h}_{i}=-\frac{\tilde{h}_{i}}{\tau}+I_{0}+I_{i}(t) .
$$

$I_{0}$ is a constant external current that is identical for all neurons. The time-dependent contribution is due to the input from other neurons,

$$
I_{i}(t)=\sum_{i} J_{i l} \alpha\left(t-t_{j}^{f}\right)
$$

As before, $I_{i j}$ is the synaptic weight representing the input amplitude. The function $a(s)$ is the typical input current caused by a presynaptic 
a)

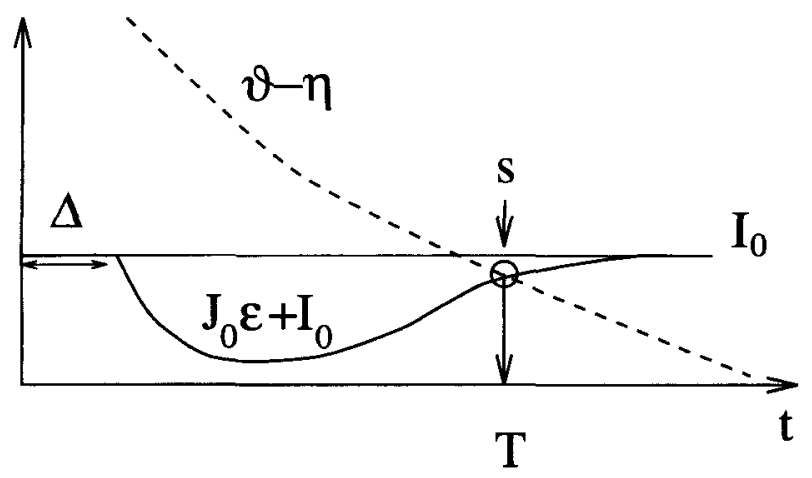

b)

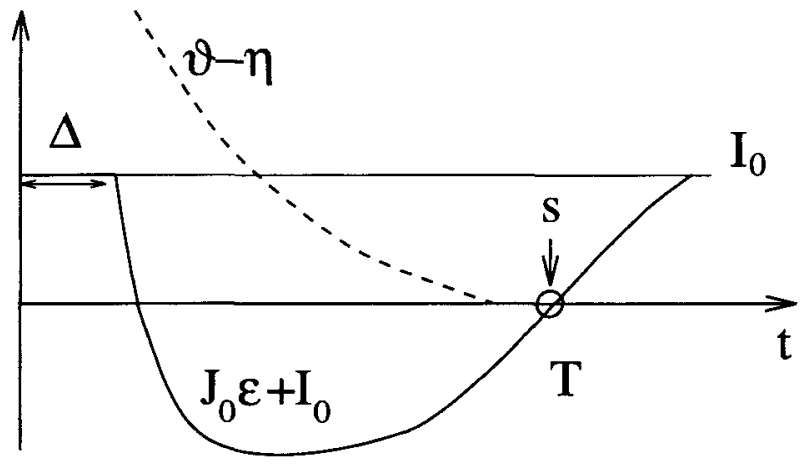

Figure 3: Geometric method: Weak (a) and strong (b) inhibitory couplings. All neurons have fired at $t=0$. The next spike occurs if $I_{0}+J_{0} \varepsilon(t)$ (solid line) crosses the decreasing effective threshold $\vartheta-\eta(t)$ (dashed line). In the case of strong and long-lasting inhibition, refractoriness has disappeared and, thus, $\eta$ already vanishes before the next spike is generated. The coherent oscillation is stable in both (a) and (b).

spike. Choices of the function $\alpha$ include $\alpha(s)=\delta(s)$, where $\delta$ is the Dirac $\delta$ function; $\alpha(s)=\delta(s-\Delta)$, where $\Delta$ is a delay; $\alpha(s)=s_{0}^{-1} \theta(s) \theta\left(s_{0}-s\right)$, for a short square pulse where $\theta(s)$ is the Heaviside unit step function; or $\alpha(s)=\left(s / \tau^{2}\right) \exp (-s / \tau)$, for a more realistic description of the synaptic input current that also obeys the pleasant normalization $\int_{0}^{\infty} \alpha(s) d s=1$. We note that the reset condition is equivalent to a current pulse $-\tilde{y} \delta(s)$ in equation 3.2. Since equation 3.2 is a linear differential equation, it can be integrated and yields

$$
\tilde{h}_{i}(t)=\sum_{f} \eta\left(t-t_{i}^{f}\right)+\sum_{j} J_{i j} \sum_{f} \varepsilon\left(t-t_{j}^{f}\right)+I_{0}\left[1-\exp \left(-\frac{t}{\tau}\right)\right]
$$


with (a prime always denoting a derivative)

$$
\eta(t)=-\tilde{j} \exp \left(-\frac{t}{\tau}\right) \Rightarrow \eta^{\prime}(t)>0
$$

and

$$
E(t)=\int_{0}^{t} d s(x) \exp \left(-\frac{t-s}{\tau}\right)
$$

The last term in equation 3.4 was adjusted so that the initial value of $\tilde{h}_{i}$ is $\hat{h}_{i}(0)=0$. We note that for $t \geqslant \tau$ the initial condition does not play any role, and the last term approaches the constant value $I_{0}$. If we define $h_{i}(t)=h_{i}(t)-I_{0}$ and $t=i-I_{0}$, we are back at equation 1.1. We would like to emphasize that the spike response model (equation 1.1) is more general than the integrate-and-fire model (equation 3.2) in that we can use arbitrary response kernels $\varepsilon$ and $\%$. A typical example of these response kernels has been presented in Figure 1.

\section{Locking}

In the following subsections, we study a coherent state of a spatially homogeneous network of $N$ neurons labeled by $1 \leq i \leq N$ and construct this network state self-consistently in such a way that the period $T$ follows directly. We first handle the existence and then turn to the stability of a coherent oscillation. The word coherent should be constantly borne in mind because it plays a key role in both the existence and the stability proof. Once a homogeneous system of spiking neurons with short-term memory behaves coherently, it cannot but oscillate. As such, oscillations are not a deep network property but simply a consequence of the connectivity and the spike dynamics of neurons. In the present context, spatial homogeneity means that all neurons are of the same type; they have identical $:$ and $/$ kernels, and have the same "gross" synaptic input: $\sum_{i} J_{i j}=J_{0}$ for all $1 \leq i \leq N$.

4.1 Existence of Coherent Solutions. In a coherent state, all neurons of the network fire synchronously and with the same period $T$. For the sake of convenience we adjust the origin $t=0$ so that regular firing occurs at $T$ with integer /. Let us assume that neurons have fired regularly in the past $t \leq 0$. More precisely, we assume that synchronous firing has occurred at $t=l T$ with $t=0 .-1 .-2 \ldots$. For $0<t<T$ the membrane potential of neuron $i$ is then given by

$$
h_{i}(t)=\sum_{i=0}^{\infty} \eta(l T+t)+\sum_{j} J_{i} \sum_{t=0}^{x} E(t T+t) .
$$


The next coherent firing should occur at time $t=T$. This means that $h_{i}(t)$ reaches the threshold $\vartheta$ at time $t=T$ and, hence, yields a self-consistency requirement for $T$,

$$
\vartheta=h_{i}(T)=\sum_{\ell=1}^{\infty}\left[\eta(\ell T)+\sum_{j} J_{i j} \varepsilon(\ell T)\right] .
$$

More precisely, $T=\inf \left\{t>0 \mid h_{i}(t)=\vartheta\right\}$. Since we have $h_{i}(t)<\vartheta$ for $t<T$, the membrane potential $h_{i}(t)$ reaches $\vartheta$ from below, and thus $h_{i}^{\prime}(T)>0$. Usually the term $\ell=1$ dominates the sum in equation 4.2 , and we end up with the simple equation

$$
\sum_{j} J_{i j} \varepsilon(T) \equiv J_{0} \varepsilon(T)=\vartheta-\eta(T) .
$$

which allows a straightforward graphic interpretation (cf. Figs. 2 and 3). Note that a delay $\Delta$ has been incorporated in $\varepsilon$. An oscillatory solution exists if the two functions $J_{0} \varepsilon(s)$ and $\vartheta-\eta(s)$ cross at some point $s^{\prime}$. If there are several crossing points, the first one (smallest $s^{\prime}$ ) gives the oscillation period $T=s^{\prime}$.

For neurons with short-term memory, that is, with $\eta(s)=\varepsilon(s)=0$ for $s \geq 2 T$, equation 4.3 is exact. For a general neuron model with adaptation, however, memory lasts longer and we have to use equation 4.2 instead of 4.3 .

4.2 Asymptotic Stability of Coherent Solutions. So far we have concentrated on the existence of coherent solutions. In the following we check whether the solutions are stable with respect to small perturbations; that is, we perform a linear stability analysis. To be specific, we consider a perturbation of the neuronal firing pattern as it occurred in the past $t \leq 0$. In the unperturbed situation, all neurons would have fired synchronously up to $t=0$, but now they do at times $\left\{\ell T+\delta_{i}(\ell) ; \ell=0,-1,-2 \ldots\right.$ and $1 \leq i \leq N\}$. We assume $\left|\delta_{i}(\ell)\right| \ll T$ since we perform a linear stability analysis. For $t>0$, the membrane potential is no longer given by equation 4.1 but by

$$
h_{i}(t)=\sum_{\ell \leq 0}\left[\eta\left(t-\ell T-\delta_{i}(\ell)\right)+\sum_{j} J_{i j} \varepsilon\left(t-\ell T-\delta_{j}(\ell)\right)\right] .
$$

At time $t=T$ the actual firing is, in general, either slightly earlier or later, and neuron $i$ fires at $T+\delta_{i}(1)$ instead of $T$. The time shift $\delta_{i}(1)$ can be found from the threshold condition $h_{i}\left(T+\delta_{i}(1)\right)=\vartheta$, given the past. We use equation 4.4, linearize with respect to all the $\delta_{i}(\ell)$ in sight, and take advantage of the unperturbed threshold condition (equation 4.2). In order to simplify the ensuing notation, we introduce the abbreviations

$$
\eta_{\ell}^{\prime}=\left.\frac{d}{d t} \eta(t)\right|_{t=\ell T}, \quad \varepsilon_{\ell}^{\prime}=\left.\frac{d}{d t} \varepsilon(t)\right|_{t=\ell T} .
$$


After a bit of algebra we then find

$$
\delta_{i}(1)=\frac{\sum_{f \geq 1}\left[\eta_{i}^{\prime} \delta_{i}(-t+1)+z_{i}^{\prime} \sum_{j} J_{i j} \lambda_{j}(-t+1)\right]}{\sum_{r \geq 1}\left(\eta_{i}^{\prime}+\xi_{i}^{\prime} \sum_{j} J_{i j}\right)} \equiv \mathbb{F}_{i}(\boldsymbol{\delta})
$$

Here $F$ is a linear map from the past $\delta$ onto the present, that is, $\left\{\delta_{i}(1) ; 1 \leq\right.$ $i \leq N\} \equiv \delta(1)$. Doing linear perturbation theory, we simply iterate $\mathbb{F}$. Proving asymptotic stability of a coherent oscillation means showing that $\lim _{k \rightarrow x} F^{k}(\delta)=0$ for an arbitrary but fixed $\delta$. We will verify below whether $\delta$ can be truly arbitrary.

Equation 4.6 is a key result of our stability analysis. Before proceeding we consider a special solution: $\lambda_{i}(-l)=\delta$ for all $i$ and $\ell$. It is an easy task to verify that $\delta_{i}(1)=\delta$ as well. That is, a uniform shift in time cannot be corrected. This is not too surprising since a system of integrate-andfire or Hodgkin-Huxley neurons or anything else that is described by a system of ordinary differential equations is unable to correct a uniform shift in time either. Mathematically, our perturbations $\delta$ therefore have to exclude a uniform time shift. Physically, the class of perturbations induced by internal "noise" or some additional stochastic input is much more restricted. Time shifts seem to be random. More precisely, we expect them to be independent, identically distributed random variables with mean zero and finite variance. If $n$ with $n \gg 1$ denotes the number of neighbors $j$ of neuron $i$, then $n^{-1} \sum_{i} \delta_{j}(-t) \approx 0$, whatever $t \geq 0$ and whatever the neuron $i$ and its surroundings, which we consider. In passing, we note that $n$ is typically of the order of a thousand or more in a vertebrate brain. Random perturbations occur all the time, but the ones stemming from the past should not blow up in the future; rather they should decay. That is why we have to iterate $F$ for a fixed argument $\delta$ and show that the result approaches zero.

The condition $\mathbb{F}^{k} \rightarrow 0$ as $k \rightarrow x$ means that the matrix $F$ should have all its eigenvalues in the open unit $\operatorname{disc}\{\lambda ;|\lambda|<1\}$. The above eigenvector $(1.1 \ldots 1)$ with eigenvalue 1 contradicts this condition. We therefore have to require that it be the only one; that is, 1 is nondegenerate (simple), its eigenvector is to be excluded, and all the other eigenvalues of $\mathbb{F}$ are less than 1 in absolute value. In passing we note that, in mathematical terms, plain instead of asymptotic stability, that is, when perturbations do not blow up but need not decay, is much cheaper. We only have to require that $|\lambda| \leq 1$ and need not worry about any further condition.

In order to interpret equation 4.6 , we assume a network where each neuron receives input from $n$ neighbors ${ }^{1}(n \gg 1)$ through homogeneous couplings $J_{i j}=J(i-j)$ where $i$ and $j$ are vectors on a two-dimensional lattice and $J(i)$ is absolutely summable, that is, $\sum_{i}|J(i)|<\infty$. There is no

\footnotetext{
'One can, but need not, think of the set of "neighbors" as a local ensemble. In the present context, it simply means the collection of presynaptic neurons.
} 
harm in assuming $\sum_{j} J_{i j}=J_{0}$, whatever $i$. Equation 4.6 is now rewritten

$$
h^{\prime} \cdot \delta_{i}(1)=\sum_{\ell \geq 0} \eta_{\ell+1} \delta_{i}(-\ell)+J_{0} \sum_{\ell \geq 0}^{k} \varepsilon_{\ell+1}^{\prime} \cdot\langle\delta(-\ell)\rangle
$$

where $h^{\prime}$, the denominator of equation 4.6, is the derivative of $h$ in equation 4.1 taken at time $T$. It is bound to be positive as the membrane potential approaches the threshold from below. Furthermore, we have introduced the mean shift $J_{0}\langle\delta(-\ell)\rangle=\sum_{j} J_{i j} \delta_{j}(-\ell)$ with $j$ ranging through the set of $n$ neighbors of $i$.

Let us assume that the mean shift $\langle\delta(-\ell)\rangle$ vanishes for all $\ell \geq 0$. If the number of neighbors $n$ is large and perturbations are random, then $\langle\delta(-\ell)\rangle \approx 0$ is a quite natural assumption. It is a simple consequence of the strong law of large numbers (Lamperti 1966; Breimann 1968). Given that $\langle\delta(-\ell)\rangle$ vanishes for all $\ell,\langle\delta(1)\rangle$ vanishes as well, a direct mathematical consequence of equation 4.7. Vanishing mean time shifts characterize a class of perturbations and thus lead to a necessary condition for a coherent oscillation to be stable. If the above argument applies, which seems fair, then this condition is also sufficient.

For the moment we simply set $\langle\delta(-\ell)\rangle=0$ and obtain from equation 4.7

$$
\delta_{i}(1)=\frac{\sum_{\ell \geq 0} \eta_{\ell+1}^{\prime} \delta_{i}(-\ell)}{\sum_{\ell \geq 0}\left(\eta_{\ell+1}^{\prime}+J_{0} \varepsilon_{\ell+1}^{\prime}\right)}
$$

This becomes truly simple for models with short-term memory where $\varepsilon(s)=\eta(s)=0$ for $s \geq 2 T$ so that the contributions $\varepsilon_{\ell}^{\prime}$ and $\eta_{\ell}^{\prime}$ can be neglected for $\ell$ beyond 1 and equation 4.8 reduces to

$$
\delta_{i}(1)=\frac{\eta_{1}^{\prime}}{\eta_{1}^{\prime}+J_{0} \varepsilon_{1}^{\prime}} \delta_{i}(0) .
$$

This is what we have used to obtain the geometric construction of Section 2. Equation 4.9 tells us two things. First, if $J_{0} \varepsilon_{1}^{\prime}>0$, then the fraction on the right is less than one, and a perturbation is bound to decrease after each spike. On the other hand, once $J_{0} \varepsilon_{1}^{\prime}<0$ is not too large in absolute value, a perturbation has to increase in time and the oscillation is unstable. The denominator in equation 4.9 is $h^{\prime}$, that is, the derivative of equation 4.1 evaluated at time $T$. Since $T$ as given by equation 4.2 determines the firing time and, on firing, the membrane potential approaches the threshold $\vartheta$ from below, $h^{\prime}$ is always positive. We end up with a dichotomy: the oscillation is stable if $J_{0} \varepsilon_{1}^{\prime}>0$ and unstable for $J_{0} \varepsilon_{1}^{\prime}<0$. Three final remarks concerning equation 4.9 are in order.

First, $J_{0} \varepsilon_{1}^{\prime}>0$ means that firing occurs while the postsynaptic potential is increasing. Second, if the neuron has forgotten its past before the next firing so that $\eta_{1}$ vanishes, then it is bound to reappear "in phase," and the oscillation is asymptotically stable. Finally, a simple geometric illustration of the stability proof can be found in Figure 4. 


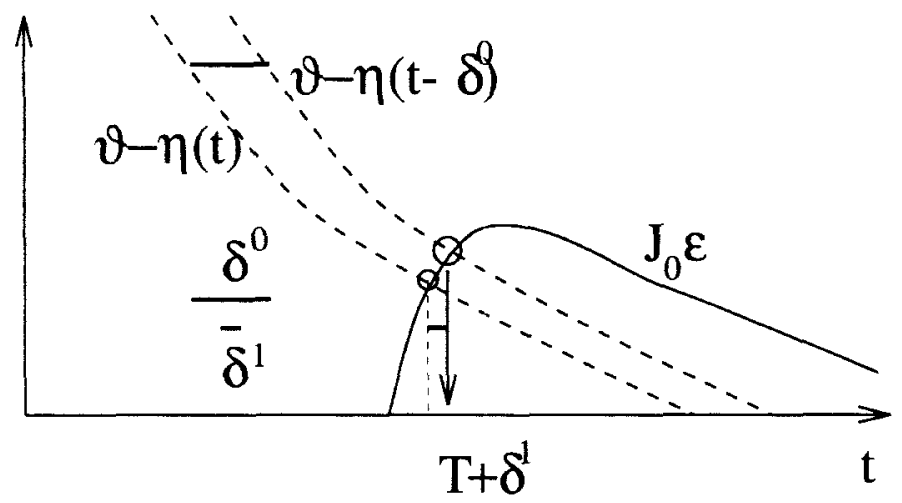

Figure 4: Geometric illustration of the locking argument. All neurons have fired at $t=0$ except for a single neuron, which is late by an amount $\delta^{0}>0$. It fires again if $J_{0}+J_{0} z(t)$ (solid line) crosses the decreasing effective threshold $y-\eta\left(t-\lambda^{0}\right)$ (dashed). The neuron is now late by an amount $\delta^{1}<\delta^{0}$ as long as the dashed lines cross the rising part of $\Sigma$. One "sees" this explicitly by comparing the projection $\delta_{1}$, indicated by an arrow, with $\delta_{0}$; both appear in the lower left-hand corner. If the dashed lines have intersections with the falling part of the response function $\Sigma$, then $\lambda^{1}>\lambda^{0}$ and the coherent oscillation is bound to be unstable.

What happens if we relax the condition of short-term memory? Neurons with a standard dynamics such as integrate-and-fire units have $\eta(s)^{\prime} \geq 0$ for all $s$ (cf. Fig. 1a). As shown in the Appendix, stability then leads to the requirement

$$
\sum_{i \geq 0} J_{0} s_{i+1}^{\prime}>0
$$

In other words, also in the general case asymptotic stability of the locked state requires that the total synaptic input be increasing at the moment when the neurons fire. This proves the necessary condition mentioned in the locking theorem. In general, one or several terms in the sum (equation 4.10) may be negative as long as the sum of all terms is positive. In fact, under the side condition of vanishing mean time shift $(n \rightarrow \infty)$, the condition (equation 4.10) is also sufficient to guarantee asymptotic stability.

The reader may wonder whether one can do without the side condition of vanishing mean shifts completely. The answer is yes, if we impose an additional constraint. We assume a standard dynamics and, in addition, require $J_{i j} \varepsilon_{\ell+1}^{\prime} \geq 0$ for all $\ell \geq 1$. In other words, we have a network of inhibitory neurons whose postsynaptic potentials decay monotonically 
or excitatory neurons whose potentials increase monotonically. Then the general stability matrix $\mathbb{F}$ as described by equation A.2 in the Appendix is a stochastic one. That is, its entries are nonnegative, and all row sums equal 1. The eigenvalues are in absolute value less than or equal to 1 ; it is indecomposable because of its special form (equation A.2); the eigenvalue $\lambda=1$ is nondegenerate; the corresponding eigenvector $(1,1, \ldots, 1)$ is to be excluded; and there is no way to reduce $\mathbb{F}$ to "cyclic form" so that all the other eigenvalues are in the open unit disc $\{\lambda ;|\lambda|<1\}$ (Horn and Johnson 1985; Gantmacher 1959). We decompose the initial vector $\delta$ with respect to the eigenvectors of $\mathbb{F}$ (Jordan decomposition) and iterate. Since there is no eigenvalue with $|\lambda|=1$ present in the decomposition, all the $\lambda^{k}$ converge to zero as $k \rightarrow \infty$. So we are done. This applies in particular to a system of leaky integrate-and-fire neurons with purely inhibitory interactions.

4.3 Nasty Counterexample. What happens if the mean time shifts do not vanish? We study a simple though somewhat academic example that serves to clarify the question: What is the response if all neurons have the same time shift $\delta(-\ell)$, which, however, is different for different $\ell$ ? That is, we assume that all neurons are synchronous but slightly aperiodic and study whether the network returns to a periodic state. The network's past clearly contradicts the requirement of vanishing mean time shift. Taking advantage of equation 4.7 , we get

$$
h^{\prime} \cdot \delta(1)=\sum_{\ell \geq 0} \eta_{\ell+1} \delta(-\ell)+J_{0} \sum_{\ell \geq 0} \varepsilon_{\ell+1}^{\prime} \delta(-\ell) .
$$

The corresponding matrix $\mathbb{F}$ (cf. the Appendix) now has the entries $\mathbb{F}_{0 \ell}=$ $\left(\eta_{\ell+1}^{\prime}+J_{0} \varepsilon_{\ell+1}^{\prime}\right) /\left(\sum_{\ell \geq 0} \eta_{\ell+1}^{\prime}+J_{0} \varepsilon_{\ell+1}^{\prime}\right)$ for $0 \leq \ell \leq \ell_{\max }-1$ in the first row and $\mathbb{F}_{\mu \nu}=\delta_{\mu, \nu+1}$ for $\mu \geq 1$. Because all row sums equal 1, there is an eigenvalue $\lambda_{1}=1$ corresponding to the eigenvector $(1,1,1, \ldots)$, a uniform time shift. We ask whether all other eigenvalues are less than 1 in absolute value. First we study a special case. Let us assume that $\eta_{\ell+1}^{\prime}+J_{0} \varepsilon_{\ell+1}^{\prime} \geq 0$ for all $\ell \geq 0$. We then arrive at a stochastic matrix and can repeat the arguments of the previous paragraph so as to conclude that all the other eigenvalues are in absolute value less than unity. Thus the neurons relax to the $T$-periodic state.

In general, the situation is more complicated since $\eta_{\ell}^{\prime}+J_{0} \varepsilon_{\ell}^{\prime}$ can be negative for some $\ell$. Take, for instance, $\ell_{\max }=2$. Then the eigenvalues are 1 (always present) and $-\mathbb{F}_{01}$. Thus, stability requires $-1<\mathbb{F}_{01}<1$. We have the boundary condition $\mathbb{F}_{00}+\mathbb{F}_{01}=1$. If $\mathbb{F}_{01}$ is outside the interval $[-1,1]$, then the neurons can remain coherent but escape from the $T$-periodic state. The state that evolves out of such an instability can be a collective bursting with the intervals between the coherent spiking of the neurons varying systematically, for example, a limit cycle of period $T_{1}+T_{2}$ where the collective interspike intervals alternate between $T_{1}$ and $T_{2}$ (cf. the Appendix, nonvanishing mean time shifts). In contrast to 
the intrinsic burster of Figure 1d, this would be a network effect. The example shows that the condition of the locking theorem is necessary but need not be sufficient as soon as the side condition of vanishing mean time shift is to be dropped-for instance, because $n$ is too small. Then additional requirements may, but need not, apply.

Stepping back for an overview, we want to isolate what requirements guarantee that equation 4.10 is both a necessary and a sufficient condition for a coherent excitation to be stable in a spatially homogeneous network of spiking neurons. There are two conditions. First, we have to restrict the network structure and require full or, at least, high connectivity. In this case, any perturbation can be separated into a uniform time shift of all neurons and a set of single-neuron time shifts with vanishing mean. We have argued that both a vanishing mean and the absence of uniform time shifts are quite natural for system-inherent perturbations of a biological network where the number of neighbors $n$ is large--the more so since coherent oscillations in the brain will last for only a finite amount of time. Second, to eliminate the-we admit, rather academicpossibility that different uniform time shifts $\delta(\theta)$ lead to an "exploding" coherent oscillation, we would have to require, say, short-term memory with $:(s)=\eta(s)=0$ for $s \geq 2 T$. Additional, especially experimental, work is needed to explore whether this requirement is really necessary or just academic.

Our results also hold in randomly diluted systems and can be extended to include variations of the parameters such as the delays (Gerstner $e$ al. 1993). A similar analysis can be used to study semicollective oscillations where the neurons spontaneously divide themselves into two or more groups of synchronous units (Gerstner and van Hemmen 1993; Gerstner 1995).

\section{Discussion and Summary}

It is time to harvest some corollaries. Before doing so we discuss the essentials of our approach. We finish the paper with a summary.

5.1 Discussion. What is the gist of what we have done? We have seen that (axonal) delays in the millisecond range are quite important. The mathematics of standard stability theory for systems with delays is very intricate (Hale 1977), not to say nasty, and the upshot, an entire function with infinitely many zeros, which all have to be located and proved to possess a negative real part, is hardly accessible to immediate analysis, if any. We have therefore proposed a more biophysical approach that directly tackles the time evolution of a perturbation: a collection of time shifts.

In Section 2, Figures 2 and 3, we have shown that coherent oscillations can exist in a system with purely excitatory interactions provided the de- 


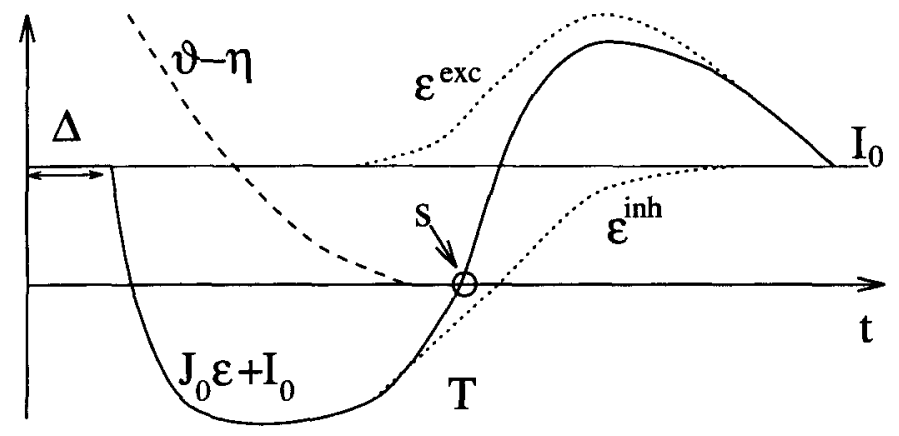

Figure 5: Geometric method: Combination of excitatory and inhibitory couplings. All neurons have fired at $t=0$. The next spike occurs once $I_{0}+J_{0} \varepsilon(t)$ (solid line) crosses the decreasing effective threshold $\vartheta-\eta(t)$ (dashed line). We assume short-range inhibition (short delay) and long-range excitation (long delay). The excitatory and inhibitory contributions are indicated by dotted lines. The sum of both yields the postsynaptic potential $J_{0} \varepsilon(t)$. The oscillation with period $T$ is stable (s) since $\eta^{\prime}=0$. A similar construction applies to the case of excitation with short delay and inhibition with long delay.

lays are long enough, that is, exceed a lower bound. On the other hand, in networks with purely inhibitory interactions, coherent oscillations are always stable, provided the delay is less than some upper bound. Most neurobiologically relevant systems, however, consist of a mixture of both excitatory and inhibitory interactions. Here we consider two models, which are, in a sense, each other's opposite. First, the inhibitory interaction is assumed to be short range and, hence, is to be associated with short delays. On the other hand, the excitatory interaction is long range and thus equipped with long delays. As is exemplified by Figure 5, here too a collective oscillation is stable. A companion model is the one with short-range excitation and long-range inhibition. One easily verifies that a similar construction shows that this setup also allows for stable coherent excitations. It is fair to summarize these results by saying that stability is determined by a subtle interplay between axonal delays, postsynaptic potentials, and refractory behavior.

Gerstner et al. (1993) and Ritz et al. (1994) have extensively studied a system with medium- or long-range excitatory interactions and a strictly local inhibition so as to represent a local but finite-range inhibitory interaction in a simplified way. "Strictly local" means that each neuron has a self-inhibitory loop with delay $\Delta$. The analytical and computational advantages are evident, but one may wonder whether this setup can be integrated into the present formalism. The answer is in the affirmative 
as one sees most easily by noticing that a self-inhibitory loop is nothing but a kind of refractory behavior and thus can be incorporated in $\eta$.

5.2 Integrate-and-Fire Neurons Revisited. Finally, it may be worthwhile to discuss a subtler, though truly academic, case that has excitatory couplings with zero delay and postsynaptic potentials with a very short rise time. Most of the integrate-and-fire models studied so far belong to this class (Mirollo and Strogatz 1990; Abbott and van Vreeswijk 1993; Tsodyks et al. 1993; Treves 1993; Usher et al. 1993). Because interactions are now instantaneous, neurons receive an excitatory postsynaptic potential as soon as one of the presynaptic neurons fires. In particular, a neuron that is late as compared to a collective oscillation experiences an extra contribution to its membrane potential (equation 4.4) of the form $\sum_{i} J_{i j}=(t)$. In other words, we have to include the $l=+1$ term in equation 4.4. If we start linearizing the shifts $\delta_{1}^{0}$ we have to take care of an extra term $s^{\prime}(0)$.

More precisely, let us assume that $\lim _{s \rightarrow 0^{+}} \mathrm{d} \varepsilon(s) / \mathrm{d} s \gg 0$. Admittedly, this is somewhat academic but illustrates the underlying locking principle quite nicely. The function $\varepsilon(s)$ is not differentiable at $s=0$ since $\varepsilon(0)=0$ for $s<0$ so $\lim _{s \rightarrow 0^{-}} \mathrm{d} s(s) / \mathrm{d} s=0$. Hence a straightforward linearization at $s=0$ is not possible. Nevertheless, we can derive analytical results if we work out the case of positive $\left(\delta_{i}^{0}>0\right)$ and negative shifts $\left(\delta_{i}^{0}<\right.$ 0) separately. Let us focus on the situation where a single neuron $i$ is too early $\left(\delta_{i}^{0}<0\right)$ and all other neurons are firing too late by a small amount $\delta^{0}>0$ so that $\left\langle\delta_{i}^{0}\right\rangle=0$. In this case, we can use equation 4.9 with (formally) $\Sigma^{\prime}<0$. Thus, $\left|\delta_{i}^{1}\right|>\left|\hat{i}_{i}^{0}\right|$ and the shift increases. On the other hand, a neuron that is late by an amount $\delta_{1}^{0}>0$ will experience an input due to not only the firings of previous cycles but also to the spikes of the very same cycle. Thus, we have to include a contribution $\propto$ $\lim _{s \rightarrow 0} \frac{d}{d s} \varepsilon(s) \gg 0$. This gives a large, positive contribution and results in a new effective $\varepsilon^{\prime} \gg 0$. Thus a neuron that is late with respect to a collective oscillation receives a strong locking signal and is immediately pulled back into synchronous firing. A neuron that fires too early, however, will fire even earlier during the next cycle (cf. Fig. 6). In principle it may happen that after several cycles, the neuron is early by nearly a full period. In this case we can consider it as being late as compared to the previous cycle, and, thus, it will be pulled into the collective oscillation. In the long run, it may happen that a collective oscillation rebuilds itself even though it is locally unstable. Since our mathematical argument is a local one and the above considerations are global, we cannot predict whether this actually happens.

Mirollo and Strogatz (1990) have shown that for some models with delayless interactions, a collective oscillation is indeed the only solution. A different form of a global argument has been put forward by Herz and Hopfield (1995; Hopfield and Herz 1995), who analyze a system 
a)

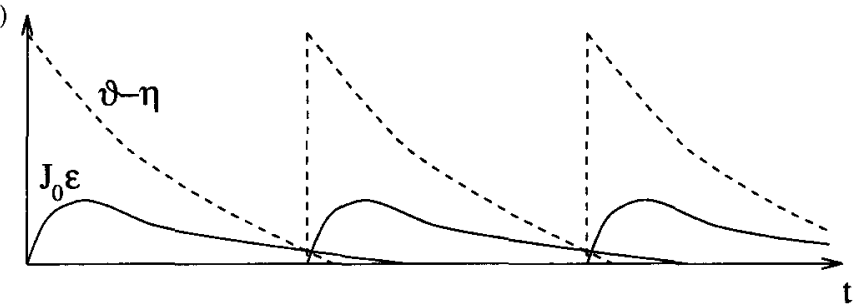

$\mathrm{T}$



Figure 6: Excitation with zero delay. (a) In a coherent oscillation, neurons would fire with a period $T$ given by the intersection of the decreasing effective threshold $\vartheta-\eta$ (dashed) and the excitation $J_{0} \varepsilon$. The whole pattern is repeated with period $T$. (b) If one of the neurons fires too early at time $t=T+\delta^{0}$ with $\delta^{0}<0$ or too late, if $\delta^{0}>0$, the decreasing threshold is shifted to the left or to the right, respectively (dotted lines). A shift to the left is increased after another period; a shift to the right is decreased. Thus, a neuron that has fired too late will be pulled back into the collective oscillation (short bar to the right of $2 T$ ), whereas a neuron that has fired too early drifts away (long bar to the left of $2 T$ ).

of nonleaky integrate-and-fire neurons with excitatory nearest-neighbor couplings $J_{i j} \geq 0$ and indicate a Lyapunov function under the conditions $\sum_{j} J_{i j}=J$ and $\sum_{i} J_{i j}=J$. Their "ingoing" condition $\sum_{j} J_{i j}=J$, whatever $i$, is directly understood once we invoke the geometric method so as to construct the solution self-consistently. As we have seen, local stability with four nearest neighbors is easily obtained, but it is hard to prove global stability. It is exactly here that a Lyapunov function pays off. It can be shown that for their nonleaky system with excitatory interaction, a whole family of solutions exists including the fully coherent state, partially synchronized states, and asynchronous firing (Herz and Hopfield 1995). 
5.3 Summary. In summary, being very conservative and, thus, dropping all side conditions, we have proved that a collective oscillation in a fully connected network of spiking neurons with standard dynamics and short-term memory $[m(s)=0$ for $s \geq 2 T$ where $T$ is the oscillation period] is an asymptotically stable solution, if firing occurs while the response due to the input from other neurons (i.e., the postsynaptic potential) is increasing. More generally, if neuronal memory lasts longer and/or if the neurons receive input from $n<N$ presynaptic neurons, then an increasing postsynaptic potential is necessary but need not be sufficient for coherent spiking. The condition is the more stringent the larger the number $n$ of interacting neighbors. In fact, we have argued that in a spatially homogeneous network with $n$ of the order of one thousand or more stability is guaranteed under the single condition of an increasing postsynaptic potential as the neurons fire.

As a consequence of our locking theorem, one can analyze existence and stability of a coherent oscillation through a purely geometric method, as sketched in Section 2. Stability holds for purely inhibitory interactions with practically arbitrary delays less than a large upper bound $\Delta<\Delta_{\max }^{\text {inh }}$ and for purely excitatory input with delays exceeding a positive lower bound $J>J_{\min }^{\mathrm{exc}}$, which depends on the network parameters. Delayless excitatory interactions are locally unstable, and all neurons that fire too early will drift away from the collective oscillation. We have also studied the case with both short-range inhibitory and long-range excitatory interaction-or the other way around-and found that coherent oscillations are abundantly present. This observation is also supported by a stability analysis of incoherent firing states. It can be shown that incoherent states are almost always unstable, and low-amplitude oscillations can form spontaneously (Abbott and van Vreeswijk 1993; Gerstner and van Hemmen 1993; Gerstner 1995). In other words, oscillations in a network of spiking neurons seem to be be omnipresent, and one has to explain why they are not found that abundantly in nature. That, maybe, is an interesting problem, which so far has not been faced.

\section{Appendix}

In this Appendix we exhibit the full mathematical structure associated with the stability matrix $\mathbb{F}$ as defined in equation 4.6. First, we discuss the general mathematical framework; then we perform the stability analysis for equation 4.8 .

General Formalism. Because of spatial homogeneity, there was no harm in assuming $\sum_{i} I_{i j}=J_{0}$, whatever $i$. We define $h^{\prime}$ to be the denominator of equation 4.6 , denote by $\mathbf{J}$ the matrix $\left(J_{i i}\right)$ and by 1 the unit matrix, 
and rewrite the equation as

$$
\boldsymbol{\delta}(1)=h^{\prime-1} \sum_{\ell \geq 0}\left[\eta_{\ell+1}^{\prime} \mathbf{1}+\varepsilon_{\ell+1}^{\prime} \mathbf{J}\right] \delta(-\ell) \equiv \sum_{\ell \geq 0} A(\ell+1) \delta(-\ell) .
$$

During the next time step, $\delta_{i}(1)$ also belongs to the past. So we are working in the Hilbert space $\mathcal{H}$, which is a direct sum of $\mathbb{R}^{N}$ with the usual inner product, labeled by $\ell$ running from 0 to $\ell_{\max }-1$. Both $\eta_{\ell}^{\prime}$ and $\epsilon_{\ell}^{\prime}$ vanish for $\ell$ beyond $\ell_{\max }$, the minimal one that does this job. In $\mathcal{H}$ we define $\mathbb{F}$ by a matrix whose elements are operators. Its first row stems from equation A.1, whose left-hand side is now called $\delta(0)$, and the other rows follow from the observation that, after one period, the present has been shifted into the past, and so on. That is, $(\mathbb{F} \delta)(-1)=\delta(0)$, $(\mathbb{F} \delta)(-2)=\delta(-1), \ldots$ so that row $\mu$ is of the form $\delta_{\mu, \nu+1} 1$. Thus we obtain the matrix

$$
\left(\begin{array}{cccccc}
A(1) & A(2) & A(3) & \cdots & A\left(\ell_{\max }-1\right) & A\left(\ell_{\max }\right) \\
\mathbf{1} & 0 & 0 & \cdots & 0 & 0 \\
0 & \mathbf{1} & 0 & \ldots & 0 & 0 \\
\ldots \ldots & \ldots \ldots & \ldots \ldots \ldots & \ldots \ldots \ldots \ldots \ldots \ldots \\
0 & 0 & 0 & \ldots & \mathbf{1} & 0
\end{array}\right)
$$

Proving asymptotic stability of a coherent oscillation means showing that $\lim _{k \rightarrow \infty} \mathbb{F}^{k}(\delta)=0$ for fixed $\delta$. It is the matrix (A.2) that has to be iterated.

Stability for Vanishing Mean Time Shifts. Here we study equation 4.8. The summations on the right-hand side have $0 \leq \ell \leq \ell_{\max }-1$. The mean time shifts vanishing, the problem becomes local, restricted to $i$, its dimension is reduced by $1 / N$ as compared to $A .2$ to $\ell_{\max }$, and we are left with a matrix whose first row has the entries $\mathbb{F}_{0 \ell}=A(\ell+1)=$ $\eta_{\ell+1}^{\prime} /\left(\sum_{\ell} \eta_{\ell+1}^{\prime}+J_{0} \sum_{\ell} \varepsilon_{\ell+1}^{\prime}\right)$, the other entries being $\mathbb{F}_{\mu \nu}=\delta_{\mu, \nu+1}$ once $\mu \geq 1$, and $0 \leq \ell, \mu, \nu \leq \ell_{\max }-1$. That is, the dimension of the problem equals $\ell_{\max }$. We have to estimate the eigenvalues of $\mathrm{F}$. In the case of short-term memory, we are left with a $1 \times 1$ matrix-the fraction in equation 4.9 . In the case of a standard dynamics, all the $\eta_{\ell}^{\prime}$ are nonnegative. Furthermore, $\sum_{\ell} \eta_{\ell+1}^{\prime}+J_{0} \sum_{\ell} \varepsilon_{\ell+1}^{\prime} \equiv h^{\prime}(T)>0$ tells us that the threshold in equation 4.2 is reached from below. Hence all the entries of $\mathbb{F}$ are nonnegative. That is, $\mathbb{F}$ is a "positive" matrix.

Positive matrices have remarkable properties (Horn and Johnson 1985; Gantmacher 1959). We list a few of them. They have a natural order: $A \geq 0$ if and only if $A_{i j} \geq 0$ for all entries of the matrix $A$, and $A \geq B$ if and only if $A-B \geq 0$. Let $\rho(A)$ denote the maximal $|\lambda|$ of the eigenvalues $\lambda$ of the matrix $A$. By good reason $\rho(A)$ is called the spectral radius. For $A \leq B$, one has $\rho(A) \leq \rho(B)$. Adopting for vectors $\mathbf{x}$ the convention $\mathbf{x}>0$ once $x_{i}>0$ for all $i$, one can show that $A \mathbf{x}=\lambda \mathbf{x}$ with $A \geq 0$ and $\mathbf{x}>0$ implies $\lambda=\rho(A)$. Moreover, if $A^{m}>0$ for some $m$ (i.e., $A$ is irreducible), then this eigenvalue is nondegenerate (simple) by a classical theorem of Perron and Frobenius, $x>0$, and, for the (noncyclic) matrix 
under consideration, it is the only eigenvalue $\lambda$ with $|\lambda|=\rho(A)$. The other eigenvalues are smaller in absolute value. As long as all the row sums are $\leq 1$, so are all the $|\lambda|$ (by the Gersgorin circle theorem [Bellman $1970]$, say). We now return to our problem.

The sum $\sum_{f} A(t)$ equals 1 if and only if $J_{0} \sum_{t} \varepsilon_{t+1}^{\prime}=0$. Then $\mathbb{F}$ is a stochastic matrix and its eigenvector $\mathbf{x}=(1.1 .1 \ldots)>0$ belongs to the eigenvalue $\rho(\mathbb{F})=1$. In passing we note that the characteristic polynomial of $\mathrm{F}$ equals

$$
\lambda^{\epsilon_{\max }}-\sum_{i=1}^{t_{\max }} A(t) \lambda^{t_{\max }-t}=0 .
$$

so that $\lambda=1$ is evidently an eigenvalue. Let $\tilde{F}$ be a matrix with $J_{0} \sum_{\varepsilon} \varepsilon_{\ell+1}^{\prime}<$ 0 or, equivalently, $\sum_{i} \dot{A}(\ell)>1$. We now allow the $A(\ell) \geq 0$ to increase from their old values belonging to $\mathbb{F}$ to their new ones associated to $\tilde{F}$. That is, we decrease some of the $z_{i+1}$ and in so doing increase some of the $A(\varphi)$. We would like to stress that we can always arrange the transformation from $\mathbb{F}$ to $\mathbb{F}$ this way.

Let us start with $A\left(\ell_{0}\right)$ and write $F(r)=F+r X$ where $X$ has a single 1 in the first row at $t=f_{0}$ and zeros everywhere else. By increasing $t$ through $i=0$ we push the eigenvalue corresponding to $\rho(\mathbb{F})=1$ through 1 at a positive rate since by perturbation theory (Kato 1966) for $k \approx 0$

$$
\rho(\mathbb{F}(h))=\rho(F)+\kappa(\mathbf{y} \cdot X \mathbf{x}) .
$$

Here $y=F^{*} \mathbf{y}$ is the eigenvector of the Hermitean adjoint matrix $\mathbb{F}^{*}$ belonging to the eigenvalue $\rho\left(\mathbb{F}^{*}\right)=1$; this matrix is also positive. The inner product $(\mathbf{y} . X \mathbf{x})=y_{0} x_{t_{0}}$ is strictly positive since $\mathbf{y}>0$, either by direct computation or from general considerations. Thus for $h>0$ we find $\rho(\mathbb{F}(t))>1$ whereas for $i<0$ we obtain $\rho(\tilde{F})<1$ as a consequence of $A \leq B$, implying $\rho(A) \leq \rho(B)$. Increasing the entries $A(\theta)$ one after the other, we arrive at the full matrix $\tilde{F}$ with $\rho(\tilde{\mathbb{F}})>1$. The corresponding eigenvector is not the uniform shift $(1.1 \ldots 1)$ and therefore cannot be excluded. This finishes the proof that $J_{0} \sum_{t} \Sigma_{i+1}^{\prime}>0$ is necessary and sufficient so as to guarantee that a coherent oscillation is asymptotically stable under perturbations with vanishing mean time shift.

Stability for Nonvanishing Mean Time Shifts. We now study a situation where all neurons have a common, nonzero, time shift $\delta(\ell)$. The evolution of the time shift is given by equation 4.11 , which reduces in the case $\ell_{\max }=2$ to

$$
\left(\begin{array}{l}
\delta(1) \\
\delta(0)
\end{array}\right)=\left(\begin{array}{cc}
F_{00} & F_{01} \\
1 & 0
\end{array}\right)\left(\begin{array}{c}
\delta(0) \\
\delta(-1)
\end{array}\right)
$$

with eigenvalues $\lambda_{0}=1$ and $\lambda_{1}=-F_{01}$. The eigenvector to $\lambda_{1}$ is $\left(-F_{01} .1\right)$.

Let us assume $F_{01}>1$ and consider a perturbation along the eigenvector corresponding to the eigenvalue $\lambda_{1}$. Specifically, we take $\delta(-1)=\delta$ 
(that is, the second last firing has been delayed by a small amount $\delta$ ) and $\delta(0)=-F_{01} \delta$ (that is, the last firing was too early by $F_{01} \delta$ ). An application of equation A.4 yields that the next firing is too late by $\delta(1)=F_{01}^{2} \delta$, the following firing is again too early by $\delta(2)=-F_{01}^{3} \delta$, and so on. It follows that, for $F_{01}>1$, the system evolves toward a bursting state where long and short intervals alternate. For $F_{01}<-1$, the delay increases monotonically as time proceeds. The present argument is a linear stability analysis and holds in the neighborhood of the oscillatory state only. It cannot predict the new limit state that the system approaches.

\section{Acknowledgments}

It is a great pleasure to Leo van Hemmen to thank Jack Cowan and the Department of Mathematics at the University of Chicago for the hospitality extended to him during his stay there, when this paper was conceived. We thank Carl van Vreeswijk (Jerusalem) for a careful reading of the manuscript and his constructive criticism, which greatly improved it.

\section{References}

Abbott, L. F. 1990. A network of oscillators. J. Phys. A: Math. Gen. 23, 3835-3859. Abbott, L. F., and van Vreeswijk, C. 1993. Asynchronous states in a network of pulse-coupled oscillators. Phys. Rev. E 48, 1483-1490.

Bauer, H. U., and Pawelzik, K. 1993. Alternating oscillatory and stochastic dynamics in a model for a neuronal assembly. Physica D 69, 380-393.

Bellman, R. 1970. Introduction to Matrix Analysis. 2d ed. McGraw-Hill, New York.

Breiman, L. 1968. Probability. Addison-Wesley, Reading, MA.

Buhmann, J. 1989. Oscillations and low firing rates in associative memory neural networks. Phys. Rev. A 40, 4145-4148.

Bush, P. C., and Douglas, R. J. 1991. Synchronization of bursting action potential discharge in a model network of neocortical neurons. Neural Comput. 3, 1930 .

Connors, B. W., and Gutnick, M. J. 1990. Intrinsic firing patterns of diverse cortical neurons. Trends in Neurosci. 13, 99-104.

Deppisch, J., Bauer, H. U., Schillen, T., König, P., Pawelzik, K., and Geisel, T. 1993. Alternating oscillatory and stochastic states in a network of spiking neurons. Network 4, 243-257.

Eckhorn, R., Bauer, R., Jordan, W., Brosch, M., Kruse, W., Munk, M., and Reitboeck, H. J. 1988. Coherent oscillations: A mechanism of feature linking in the visual cortex? Biol. Cybern. 60, 121-130.

Eckhorn, R., Frien, A., Bauer, R., Woelbern, T., and Kehr, H. 1993. High frequency $(60-90 \mathrm{~Hz})$ oscillations in primary visual cortex of awake monkey. NeuroReport 4, 243-246. 
Engel, A. K., König, P., and Singer, W. 1991a. Direct physiological evidence for scene segmentation by temporal coding. Proc. Natl. Acad. Sci. USA, 88, 9136-9140.

Engel, A. K., König, P., Kreiter, A. K., and Singer, W. 1991b. Interhemispheric synchronization of oscillatory neural responses in cat visual cortex. Science 252, 1177-1179.

Engel, A. K., König, P., Kreiter, A. K., Schillen, T. B., and Singer, W. 1992. Temporal encoding in the visual cortex: New vistas on integration in the nervous system. Trends Neurosc. 15, 218-226.

Ernst, U., Pawelzik, K., and Geisel, T. 1995. Synchronization induced by temporal delays in pulse-coupled oscillators. Phys. Rev. Lett. 74, 1570-1573.

Gantmacher, F. R. 1959. Matrix Theory, Vol. 2. Chelsea, New York.

Gerstner, W. 1991. Associative memory in a network of "biological" neurons. In Advances in Neural Information Processing Systems 3, R. P. Lippmann, J. E. Moody, and D. S. Touretzky, eds., pp. 84-90, Morgan Kaufmann, San Mateo, CA.

Gerstner, W. 1995. Time structure of the activity in neural network models. Phys. Rez: E 51, 738-758.

Gerstner, W., and van Hemmen, J. L. 1992. Associative memory in a network of "spiking" neurons. Network 3, 139-164.

Gerstner, W., and van Hemmen, J. L. 1993. Coherence and incoherence in a globally coupled ensemble of pulse emitting units. Plyys. Rec'. Lett. 71, 312315.

Gerstner, W., Ritz, R, and van Hemmen, J. L. 1993. A biologically motivated and analytically soluble model of collective oscillations in the cortex: I. Theory of weak locking. Biol. Cybern. 68, 363-374.

Golomb, D., Hansel, D., Shraiman, B., and Sompolinsky, H. 1992. Clustering in globally coupled phase oscillators. Phys. Ret. A 45, 3516-3530.

Gray, C. M. 1994. Synchronous oscillations in neuronal systems: Mechanisms and functions /. Comput. Neurosci. 1, 11-38.

Gray, C. M., and Singer, W. 1989. Stimulus-specific neuronal oscillations in orientation columns of cat visual cortex. Proc. Natl. Acad. Sci. USA 86, 16981702.

Gray, C. M., König, P., Engel, A. K., and Singer, W. 1989. Oscillatory responses in cat visual cortex exhibit inter-columnar synchronization which reflects global stimulus properties. Nature 338, 334-337.

Hale, J. K. 1977. The'ory of Functional Differential Equations. Springer, New York.

Hansel, D., Mato, G., and Meunier, C. 1995. Synchronization in excitatory neural networks. Netural Comput. 7, 307-337.

Herz, A. V. M., and Hopfield, J. J. 1995. Earthquake cycles and neural reverberations: Collective oscillations in systems with pulse-coupled threshold elements. Phys. Rec. Lett. 75, 1222-1225.

Hopfield, J. J., and Herz, A. V. M. 1995. Rapid local synchronization of action potentials: Towards computation with coupled integrate-and-fire neurons. Proc. Natl. Acad. Sci. USA 92, 6655-6659.

Horn, R. A., and Johnson, C. R. 1985. Matrix Analysis. Cambridge University Press, Cambridge. 
Kato, T. 1966. Perturbation Theory for Linear Operators. Springer, New York.

Kistler, W., Gerstner, W., and van Hemmen, J. L. 1996. Reduction of the Hodgkin-Huxley equations to an optimized threshold model. Submitted to Neural Comput.

König, P., and Schillen, T. B. 1991. Stimulus-dependent assembly formation of oscillatory responses: I. Synchronization. Neural Comput. 3, 155-166.

Kuramoto, Y. 1991. Collective synchronization of pulse-coupled oscillators and excitable units. Physica D 50, 15-30.

Lamperti, J. 1966. Probability. Benjamin, New York.

Lytton, W. W., and Sejnowski, T. J. 1991. Simulations of cortical pyramidal neurons synchronized by inhibitory interneurons. J. Neurophysiol. 66, 10591079.

von der Malsburg, C. 1994. The correlation theory of brain function. In Models of Neural Networks II, E. Domany, J. L. van Hemmen, and K. Schulten, eds., pp. 95-119, Springer, New York (reprint of the unpublished 1981 paper).

von der Malsburg, C., and Buhmann, J. 1992. Sensory segmentation with coupled neural oscillators. Biol. Cybern. 67, 233-242.

von der Malsburg, C., and Schneider, W. 1986. A neural cocktail-party processor. Biol. Cybern. 54, 29-40.

Mirollo, R. E., and Strogatz, S. H. 1990. Synchronization of pulse coupled biological oscillators. SIAM J. Appl. Math. 50, 1645-1662.

Niebur, E., Kammen, D. M., Koch, C., Rudermann, D., and Schuster, H. G. 1991. Phase-coupling in two dimensional networks of interacting oscillators. In Advances in Neural Information Processing Systems 3, R. P. Lippmann, J. E. Moody, and D. S. Touretzky, eds., pp. 123-127, Morgan Kaufmann, San Mateo, CA.

Nischwitz, A., and Glünder, H. 1995. Local lateral inhibition: A key to spike synchronization? Biol. Cybern. 73, 389-400.

Ritz, R., Gerstner, W., and van Hemmen, J. L. 1994. Associative binding and segregation in a network of spiking neurons. In Models of Neural Networks II, E. Domany, J. L. van Hemmen, and K. Schulten, eds., pp. 175-219, Springer, New York.

Schillen, T. B., and König, P. 1991. Stimulus-dependent assembly formation of oscillatory responses: II. Desynchronization. Neural Comput. 3, 167-178.

Schuster, H. G., and Wagner, P. 1990a. A model for neuronal oscillations in the visual cortex: 1 . Mean-field theory and derivation of the phase equations. Biol. Cybern. 64, 77-82.

Schuster, H. G., and Wagner, P. 1990b. A model for neuronal oscillations in the visual cortex: 2 . Phase description and feature dependent synchronization. Biol. Cybern. 64, 83-85.

Singer, W. 1994. The role of synchrony in neocortical processing and synaptic plasticity. In Models of Neural Networks II, E. Domany, J. L. van Hemmen, and K. Schulten, eds., pp. 141-173, Springer, New York.

Skinner, F. K., Kopell, N., and Marder, E. 1994. Mechanisms for oscillation and frequency control in reciprocally inhibitory model neural networks. I. Comput. Neurosci. 1, 69-87. 
Sompolinsky, H., Golomb, D., and Kleinfeld, D. 1990. Global processing of visual stimuli in a neural network of coupled oscillators. Proc. Natl. Acad. Sci. USA 87, 7200-7204.

Treves, A. 1993. Mean-field analysis of neuronal spike dynamics. Network 4, 259-284.

Tsodyks, M., Mitkov, I., and Sompolinsky, H. 1993. Patterns of synchrony in inhomogeneous networks of oscillators with pulse interaction. Phys. Rev. Lett. 71, 1281-1283.

Usher, M., Schuster, H. G., and Niebur, E. 1993. Dynamics of populations of integrate-and-fire neurons, partial synchronization, and memory. Neural Comput. 5, 570-586.

van Vreeswijk, C., Abbott, L. F., and Ermentrout, G. B. 1994. When inhibition not excitation synchronizes neural firing. I. Comp. Neurosc. 1, 313-321.

Received August 31, 1995; accepted April 9, 1996. 